\title{
STATISTICAL ANALYSIS OF LOCAL COMPOSITION AND LUMINESCENCE IN InGaN GROWN BY MOLECULAR BEAM EPITAXY
}

\author{
S. Einfeldt, * T. Böttcher,* D. Hommel,* H. Selke,** P. L. Ryder,** \\ F. Bertram, ${ }^{* * *}$ T. Riemann, ${ }^{* * *}$ D. Rudloff, $* * *$ J. Christen $* * *$ \\ * Institute of Solid State Physics, University of Bremen, \\ PO Box 330440, 28334 Bremen, Germany \\ ** Institute of Materials Physics and Structural Research, University of Bremen, \\ PO Box 330440, 28334 Bremen, Germany \\ *** Institute of Experimental Physics, University of Magdeburg, \\ PO Box 4120, 39016 Magdeburg, Germany
}

Cite this article as: MRS Internet J. Nitride Semicond. Res. 4S1, G3.33(1999)

\begin{abstract}
$\underline{\text { Abstract }}$
InGaN layers grown by molecular beam epitaxy are investigated in terms of their compositional homogeneity using transmission electron microscopy and cathodoluminescence spectroscopy performed with high spatial resolution. Strong fluctuations of the indium content were found in bulklike layers, which could be partially reduced by modulating the indium flux during growth, i. e. by nominally growing a short period $\mathrm{GaN} / \mathrm{InGaN}$ superlattice. For indium compositions above $x \cong 0.1$ this approach fails. Strained InGaN in quantum wells exhibits lateral fluctuations on an atomic scale and on a scale of several hundred nanometers. The results are discussed in view of the origin of inhomogeneous indium incorporation.
\end{abstract}

\section{$\underline{\text { Introduction }}$}

Although the ternary alloy InGaN is established as the active material in optoelectronic devices operating in the short wavelength region, its epitaxial growth raises significant problems making it a subject of numerous investigations. The large lattice mismatch between $\mathrm{InN}$ and $\mathrm{GaN}$ is the reason for a miscibility gap in the phase diagram of InGaN, which could result in a demixing into phases of different composition during growth [1,2]. Moreover, the binding energy is significantly lower for InN compared to $\mathrm{GaN}$, such that the thermal decomposition of InGaN causing the formation of indium droplets on the growth surface is a possible source of lateral variations of the composition [3]. Furthermore, compositional fluctuations arise from lattice relaxation of strained InGaN films on GaN due to V-shaped defects [4] and the composition pulling effect [5].

Direct evidence of compositional fluctuations in InGaN bulk-like layers and quantum well structures was given by spatially resolved measurements of optical and structural properties utilizing cathodoluminescence (CL) spectroscopy [6] and transmission electron microscopy (TEM) [7,8]. In this paper the attempt is started to distinguish between possible reasons for these fluctuations is with the object to draw conclusions for the optimization of expitaxial growth. Therefore, InGaN/GaN heterostructures were grown under different conditions and investigated by CL and TEM.

\section{$\underline{\text { Experimental }}$}

Epitaxial growth was performed in a standard molecular beam epitaxy (MBE) system equipped with Knudsen cells for gallium and indium and a radio frequency plasma source (EPI Unibulb) operated with pure nitrogen gas. C-plane sapphire substrates were first nitridated using the nitrogen plasma 
followed by the growth of a $\mathrm{GaN}$ layer of variable thickness. Its growth temperature was $650^{\circ} \mathrm{C}$ for small layer thicknesses of $30 \mathrm{~nm}, 750^{\circ} \mathrm{C}$ for thicker layers and $830^{\circ} \mathrm{C}$ for cladding layers of quantum wells. InGaN was always grown at $650^{\circ} \mathrm{C}$.

Cross-sectional specimens for TEM were prepared by mechanical grinding to about $5 \mu \mathrm{m}$ thickness followed by xenon ion milling at $5 \mathrm{kV}$. TEM experiments were carried out on a Philips EM420 operating at $120 \mathrm{kV}$ and equipped with a scanning TEM system and an energy-dispersive Xray (EDX) spectrometer. Using a $10 \mathrm{~nm}$ electron probe the local chemical composition was deduced from the intensity ratio of the indium L- and gallium K-lines applying the standardless thin film method. Frequency distributions of the indium content were typically determined from EDX mappings of $256 \times 200$ points over a lateral length of $3 \mu \mathrm{m}$. Since the EDX mappings contain significant noise due to a limited integration time for each measured point, the real magnitude of compositional fluctuations is overestimated using the width of these distributions. Digital analysis of high resolution TEM lattice images (DALI) was applied to obtain mappings of the local lattice parameter $c$ in InGaN/GaN quantum wells [9]. Assuming a Poisson ratio of 0.51 and neglecting relaxation of the fully strained wells the lattice parameters were converted into a local indium content. Since only relative variations of the indium content will be discussed, possible deviations from these assumptions are not relevant.

CL was measured in plan-view configuration usually at $5 \mathrm{~K}$ using a modified, high-resolution scanning electron microscope. Scanning the focused electron beam over an area of $(2 \mu \mathrm{m} \times 3 \mu \mathrm{m})$ to $(13 \mu \mathrm{m} \times 20 \mu \mathrm{m})$, a local spectrum was recorded at each of the $256 \times 200$ points. Typical changes of local spectra were found on a lateral length scale of a few hundred nanometer for all samples under investigation. The set of more than 50.000 local spectra was used to derive an integral (averaged) spectrum and a frequency distribution of the peak energies in the local spectra.

\section{$\underline{\text { Results }}$}

Figure 1 illustrates the variation of the local composition in a typical bulk-like InGaN layer of $1 \mu \mathrm{m}$ thickness on a thin GaN layer which in the following is referred to as conventionally grown. Based on $\mathrm{X}$-ray diffraction (XRD) measurements the mean indium mole fraction of this samples amounts to $x=$ 0.08. In ref. 10 the laterally averaged indium content was shown to decrease up to a layer thickness of $300 \mathrm{~nm}$ and to remain constant up close to the surface. This fact is confirmed by the two frequency distributions of the local indium content shown in Figure 1a, whose maxima are clearly shifted against each other. The large width of both distributions reflects the strong lateral fluctuation of the composition discussed in ref. 6. However, a quantization of the fluctuations should not be deduced from Figure 1a due to significant noise in the used EDX mapping. Since the band gap of InGaN naturally depends on the indium content, the energy of the near-band gap luminescence changes laterally across the sample which was shown in ref. 6 as well. Thus the frequency distribution of peak energies of the local CL spectra presented in Figure $1 \mathrm{~b}$ is closely related to the local indium distribution. Obviously the distribution is broad and asymmetric as could be expected from Figure 1a. The superposition of all local spectra results in a large full width at half maximum (FWHM) of $0.35 \mathrm{eV}$ for the peak in the integral spectrum shown in Figure $1 \mathrm{~b}$.

In the following possible origins of fluctuations will be discussed. The first parameter under consideration is the crystal perfection of InGaN. Extended defects are known to act as points of local lattice relaxation and by this can increase the local indium content without increasing strain energy. The defect density in InGaN is limited by the crystal perfection of the GaN grown below which improves with its layer thickness. Therefore, InGaN was grown under comparable conditions on GaN buffer layers of up to $1 \mu \mathrm{m}$ thickness. Without showing results in this paper similar lateral fluctuations were found even for the thickest GaN buffer layers. In conclusion, if defects are the relevant source for compositional fluctuations, MBE growth of thick GaN layers does not sufficiently reduce their density to effectively improve the compositional homogeneity of InGaN. 

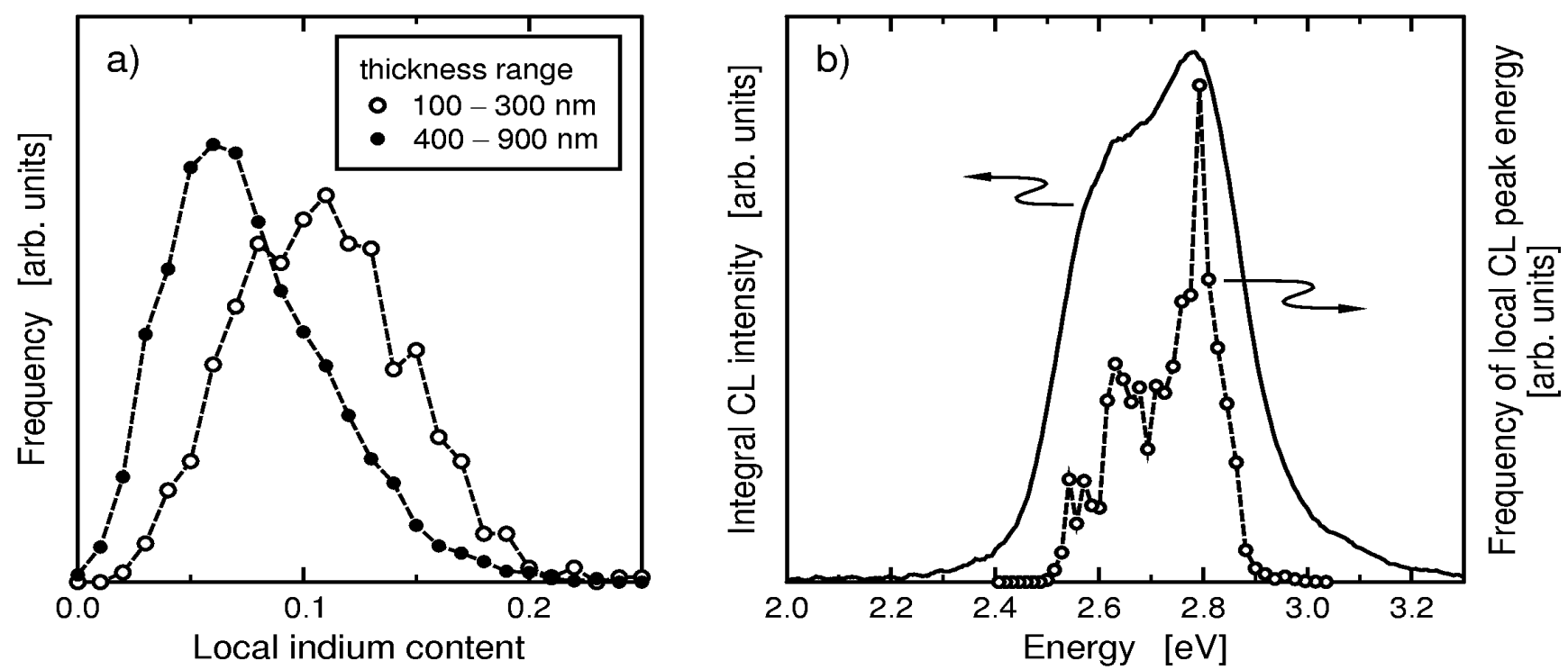

Figure 1: Conventionally grown $\mathrm{In}_{0.08} \mathrm{Ga}_{0.92} \mathrm{~N}(1000 \mathrm{~nm})$ on $\mathrm{GaN}(30 \mathrm{~nm})$ : a) Frequency distribution of the local indium content as determined by EDX analysis averaged over InGaN layer thicknesses of 100-300 and 400-900 nm. b) Integral CL spectrum and frequency distribution of the local CL peak energy.

A second approach discussing fluctuations is to take the formation of microscopic indium droplets on the growth surface into consideration. Those droplets would lead to a lateral variation of the indium content but could also act as a drain for impinging indium atoms resulting in a variation of the mean indium content with layer thickness as described above. Johnson et al. first suggested to oppose droplet formation by modulating the indium flux during growth [3]. During GaN growth of a nominal InGaN/GaN superlattice with a period of about one or two monolayers, excess indium on the surface is supposed to be incorporated into the epitaxial film. In this paper this growth regime will be referred to as modulated growth. Results of two modulated grown InGaN layers differing in thickness $(1.0$ and $0.3 \mu \mathrm{m})$ and mean indium content $(0.13$ and 0.05 according to XRD measurements) are presented in Figure 2. Since no hints for the excistence of a superlattice were found by XRD or TEM measurements, a complete intermixing of InGaN and GaN probably occurs during growth. The mean composition of sample $\mathrm{A}$ is constant along growth direction as confirmed by EDX mappings, whereas this remains unclear for sample B as the low indium content makes appropriate measurements impossible. Although the indium distribution in Figure 2a still indicates the presence of lateral fluctuations, it appears to be less asymmetric than in the conventionally grown sample. The asymmetry for the last sample is a consequence of few extremely indium rich columns observed in the EDX mapping published in ref 8. Similar structures are not found in modulated grown samples. Moreover, EDX spot measurements carried out with long integration times at various points of the sample show the lateral variation of the indium content to be successfully reduced.

Figure $2 b$ shows CL results of the modulated grown layers. In agreement with the distribution from Figure 2a, the asymmetry of the integral spectra as well as of the frequency distributions of the local peak energies is less pronounced compared to the conventionally grown sample. Moreover, the distinct multimodal character of the local peak energy distribution shown in Figure $1 \mathrm{~b}$ can not be observed. However, considering sample A the local CL peak energy varies to the same extent as for the conventionally grown sample, resulting in an integral peak with a similar FWHM of $0.34 \mathrm{eV}$. In contrast, the value of the same FWHM for sample B amounts to only $0.19 \mathrm{eV}$ and the lateral homogeneity is obviously improved as indicated by the very narrow frequency distribution of the local 

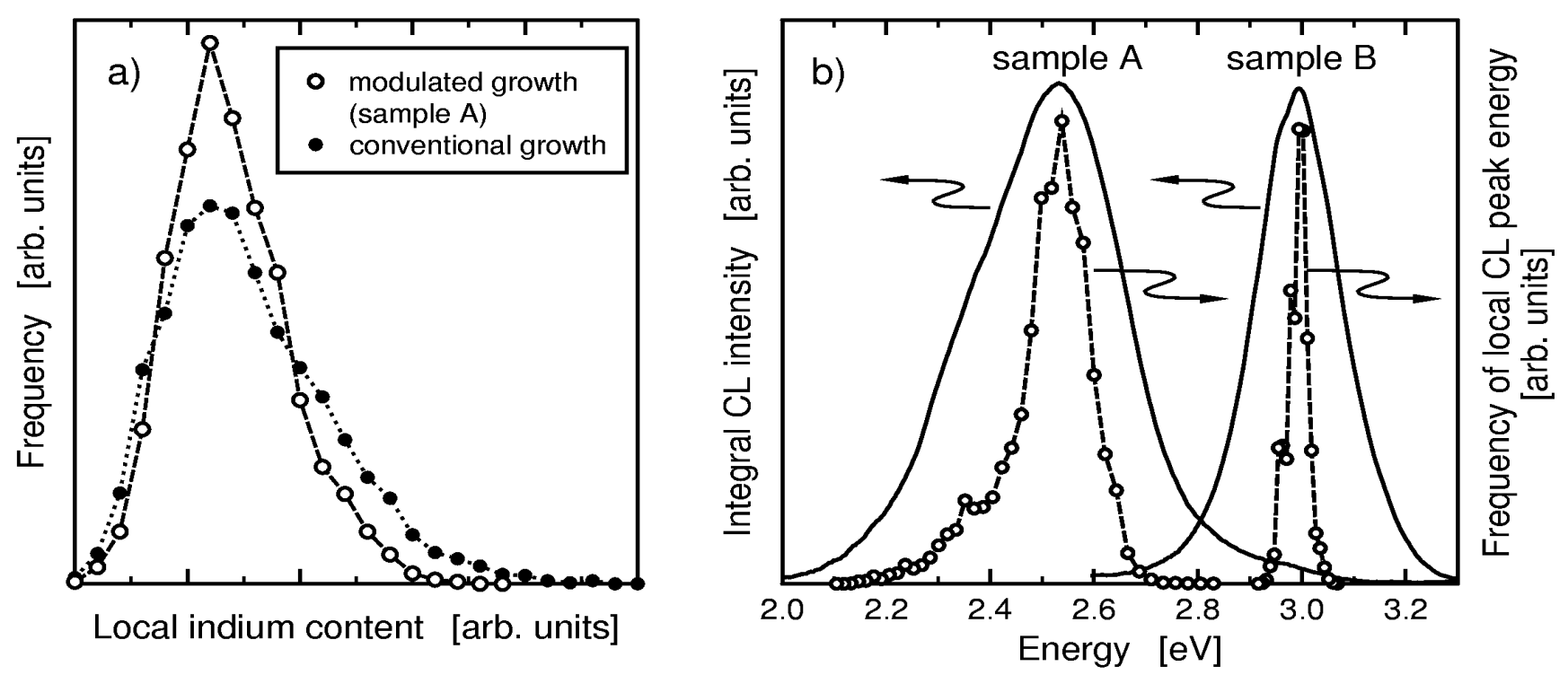

Figure 2: Modulated grown $\operatorname{In}_{x} \mathrm{Ga}_{1-x} \mathrm{~N}$. Sample $\mathrm{A}(x=0.13): 940 \times(0.7 \mathrm{~nm} \mathrm{InGaN} / 0.3 \mathrm{~nm} \mathrm{GaN})$ on $200 \mathrm{~nm}$ GaN. Sample B $(x=0.05): 300 \times(0.5 \mathrm{~nm} \mathrm{InGaN} / 0.5 \mathrm{~nm} \mathrm{GaN})$ on $30 \mathrm{~nm} \mathrm{GaN}$. a) Frequency distribution of the local indium content as determined by EDX analysis for sample A and the reference sample of Figure 1 averaged over InGaN layer thicknesses of 400-900 nm. b) Integral CL spectra and frequency distributions of the local CL peak energy for samples A and B.

CL peak energies. This has to be a consequence of the lower mean indium content and/or the smaller InGaN layer thickness of sample B.

Based on further CL experiments the extent of fluctuations in unstrained conventionally grown samples does not significantly change when the layer thickness is increased from 0.3 to $1 \mu \mathrm{m}$. Moreover, conventionally grown samples with mean indium contents comparably low as that of sample B show an integral luminescence peak which is broader by a factor of two and red-shifted by about $0.2 \mathrm{eV}$. This suggests that the improved homogeneity of sample B is a result of combining both the modulated growth technique and the low mean indium content. For higher indium contents as for sample A the concept of modulated growth fails. Due to the high indium fluxes required in this case the amount of indium on the surface is probably too high to be incorporated into the layer during the short period of nominal GaN growth. However, thermodynamically driven demixing as expected by the phase diagram of InGaN for higher indium contents could be an explanation as well.

A parameter worth to discuss in terms of fluctuations in InGaN is the strain state of the material. Karpov calculated phase diagrams for relaxed and strained $\mathrm{InGaN}$, indicating the miscibility gap to extend into regions of relevant MBE growth temperatures and interesting indium contents up to 0.2 only in the case of relaxed material [2]. Thus one may expect significantly lower lateral fluctuations for fully strained InGaN layers as in InGaN/GaN quantum wells. Figure 3 summarizes the results for a multiquantum well (MQW) structure with conventionally grown InGaN wells containing roughly $10 \%$ indium. High resolution TEM images confirm that the wells are fully strained. The mapping in Figure 3a generated by DALI clearly shows a lateral variation of the local lattice parameter within the wells. The fluctuations are irregular but marked by pronounced dot-like structures with diameters of $5 \mathrm{~nm}$ or less which are not correlated to extended defects. Similar structures were already reported by others [7]. Figure $3 b$ shows the broad frequency distribution of the local indium content in the uppermost quantum well marked by several maxima. Despite the relatively small number of lattice points analysed for Figure $3 b$, the presented distribution is representative because analogous distributions for other wells look similar. 

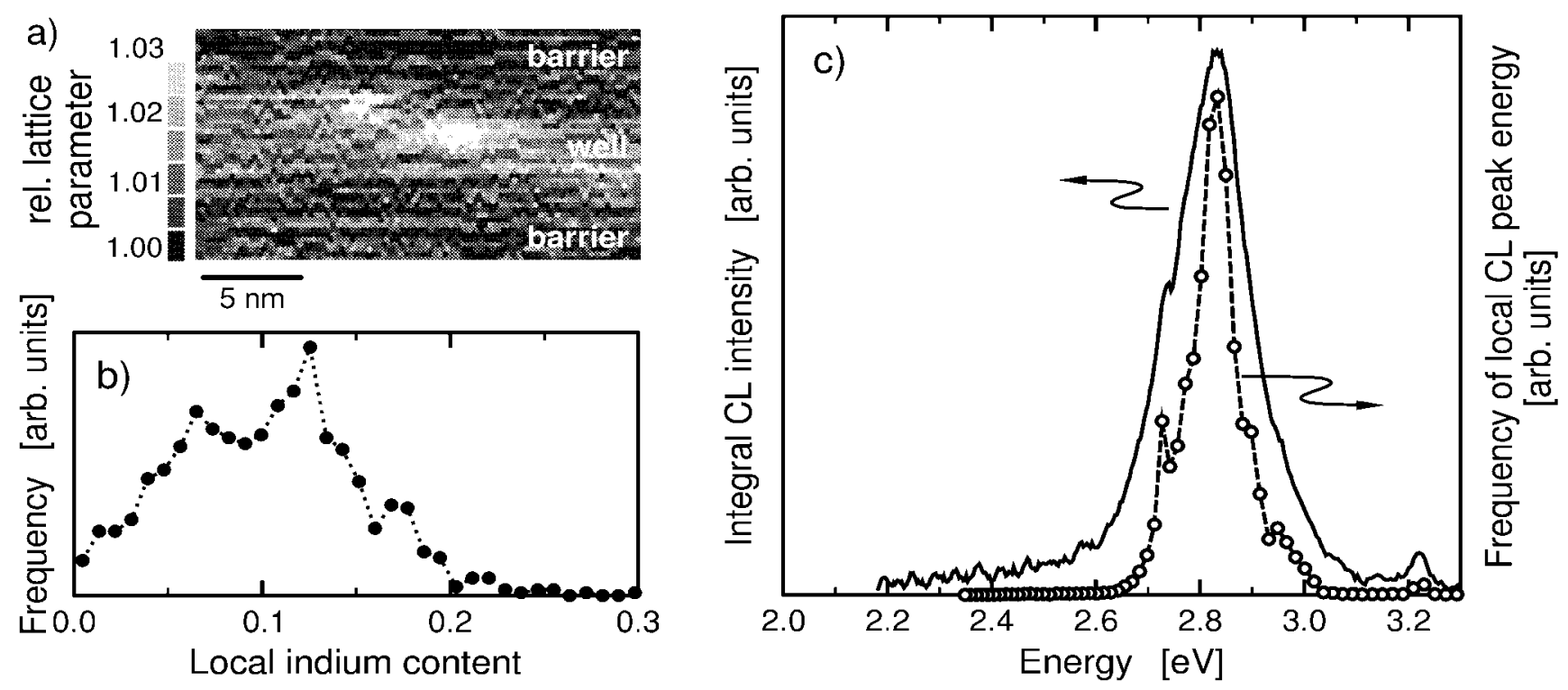

Figure 3: GaN/InGaN MQW: GaN (200 nm) / $5 \times(5 \mathrm{~nm}$ InGaN / $10 \mathrm{~nm} \mathrm{GaN}) / \mathrm{GaN}(300 \mathrm{~nm}) /$ sapphire. a) Mapping of the local lattice parameter as determined by DALI. b) Frequency distribution of the local indium content in a single well. c) Integral CL spectrum and frequency distribution of the local CL peak energy.

Due to a different lateral extension of the EDX and DALI mappings the distribution in Figure $3 \mathrm{~b}$ can not be simply compared to those in Figure 1a and 2a. However, Figure 3a prooves that variations of the indium content were not completly be avoided even in strained InGaN. Consequently these short-range fluctuations are rather related to the growth kinetics than to thermodynamical reasons. Also, it can not be excluded that fluctuations are caused by diffusion during growth of the final $\mathrm{GaN}$ capping layer carried out at higher temperatures compared to the well region.

Luminescence characteristics of the MQW are shown in Figure 3c. Due to the large well width they can be discussed without considering quantization effects. The FWHM of $0.18 \mathrm{eV}$ for the integral CL peak is smaller than that extracted from Figure 1 corresponding to a thick layer grown under a similar indium flux. The frequency distribution of the local peak energy is narrower and less asymmetric as well. Thus the uniformity on a lateral scale accessible by CL is improved by the growth of strained quantum wells. However, mappings of the peak energy for this sample look similar to those of bulk-like samples published in ref. 6. They clearly show fluctuations on a scale of a few hundred nanometer roughly corresponding to the grain size of GaN. The point is how to reconcile this to the discussed dot-like structures on a nanometer scale. Excited carriers will relax into local potential minima due to diffusion resulting in a nearly constant recombination energy within their diffusion length as observed in CL mappings. It is not clear whether the dot-like structures observed by DALI form the dominant potential minima acting as preferred recombination centers. Assuming this case, the frequency distribution of mean dot composition obviously has to vary from grain to grain to be not inconsistent with the CL mappings. Furthermore, similar dot-like structures should occur in bulk-like samples as well. The difference between CL spectra in Figure $1 b$ and $3 c$ could arise from the undoubtedly different defect structures in these layers leading to a different impact of the dots on the recombination process.

Comparing the integral spectra of the MQW and the modulated grown sample B, similar values for the FWHM are surprisingly found although sample B is more homgeneous in the sense that its local peaks are broad but located at the same spectral position. Considering the higher density of extended defects in sample B compared to the MQW structure the broadening of the local spectra for sample B 
is believed to result from short-range fluctuations of the composition and/or of the defect distribution which are not resolved by CL due to its limited resolution.

\section{$\underline{\text { Conclusions }}$}

Strong compositional fluctuations in $\mathrm{MBE}$ grown InGaN bulk-like layers can be reduced by a modulated growth technique in case the indium content is sufficiently low. This fact is in agreement with the model of droplet formation on the growth surface assumed to be one possible source of inhomogeneities in the material. For higher indium concentrations the strain relaxation seems not to be the dominant factor for fluctuations because even strained quantum wells show fluctuations on the scale of the well width as well as of the $\mathrm{GaN}$ grain size. To understand these fluctuations quantum wells containing different amounts of indium and grown with different growth techniques have to be compared. Moreover, defects or dot-like structures should be identified as dominant recombination centers for which the growth on GaN with a significantly reduced dislocation density is a worthwhile approach.

\section{$\underline{\text { Acknowledgment }}$}

This work was supported by the Volkswagen-Stiftung, contract no. I/74 452.

\section{$\underline{\text { References }}$}

[1] I-hsiu Ho and G. B. Stringfellow, J. Cryst. Growth 178, 1 (1997).

[2] S. Yu. Karpov, Internet J. Nitride Semicond. Res. 3, 16 (1998).

[3] M. A. L. Johnson, W. C. Highes, W. H. Rowland, Jr., J. F. Schetzina, M. Leonard, H. S. Kong, J. A. D. Edmond and J. Zavada, J. Cryst. Growth 175/6, 72 (1997).

[4] B. Jahnen, M. Albrecht, W. Dorsch, S. Christiansen, H. Strunk, D. Hanser and Robert F. Davis, MRS Internet J. Nitride Semicond. Res. 3, 39 (1998).

[5] K. Hiramatsu, Y. Kawaguchi, M. Shimizu, N. Sawaki, T. Zheleva, Robert F. Davis, H. Tsuda, W. Taki, N. Kuwano and K. Oki, MRS Internet J. Nitride Semicond. Res. 2, 6 (1997).

[6] H. Selke, P. L. Ryder, T. Böttcher, S. Einfeldt, V. Kirchner, D. Hommel, F. Bertram, T. Riemann and J. Christen, submitted to J. Cryst. Growth.

[7] Y. Narakuwa, Y. Kawakami, M. Funato, S. Fujita, S. Fujita and S. Nakamura, Appl. Phys. Lett. 70, 981 (1997).

[8] H. Selke, M. Amirsawadkouhi, P. L. Ryder, T. Böttcher, S. Einfeldt, D. Hommel, F. Bertram, J. Christen, to be published in Mat. Sci. Eng. B.

[9] A. Rosenauer, S. Kaiser, T. Reisinger, J. Zweck, W. Gebhardt and D. Gerthsen, Optik 102, 63 (1996).

[10] T. Böttcher, S. Einfeldt, V. Kirchner, S. Figge, H. Heinke, D. Hommel, H. Selke and P. L. Ryder, Appl. Phys. Lett. 73, 3232 (1998). 